\title{
Use, Misuse, and Abuse of Apparent Diffusion Coefficients
}

\author{
DENIS S. GREBENKOV
}

Laboratoire de Physique de la Matière Condensée, CNRS, Ecole Polytechnique, Palaiseau F-91128, France

\begin{abstract}
The notion of effective, time-dependent or, equivalently, apparent diffusion coefficient $(A D C)$ and its use for characterization of porous media are revisited. It is argued that the dynamic ADC, quantifying the mean-square displacement of spin-bearing particles, should not be confused with its counterpart measured by a pulsed gradient spin echo technique. The former is an intrinsic characteristic of the medium, independent of the applied magnetic field. In contrast, the spin-echo ADC depends on the experimental setup (e.g., gradient intensity and temporal profile), raising potential ambiguities in the interpretation of diffusion-weighted measurements, which may be strongly misleading when the Gaussian phase approximation (GPA) does not hold. The oversimplified use of a single $b$-value is criticized. Several fitting models beyond the GPA are discussed. (C) 2010 Wiley Periodicals, Inc. Concepts Magn Reson Part A 36A: 24-35, 2010.
\end{abstract}

KEY WORDS: ADC; diffusion-weighted imaging; restricted diffusion; NMR; PGSE; geometry

\section{INTRODUCTION}

\section{Unrestricted Normal Diffusion}

Diffusion of spin-bearing particles in a complex medium is a common process for various fields, from physiology to material sciences $(1,2)$. A typical example is diffusion of water molecules in sedimentary rocks or biological tissues. Lacking precise

Received 3 April 2009; revised 30 September 2009; accepted 27 October 2009

Correspondence to: Denis Grebenkov. E-mail: denis.grebenkov@ polytechnique.edu;

Concepts in Magnetic Resonance Part A, Vol. 36A(1) 24-35 (2010)

Published online in Wiley InterScience (www.interscience.wiley. com). DOI 10.1002/cmr.a.20152

(c) 2010 Wiley Periodicals, Inc. description of a huge number of water molecules in a liquid phase, the position $\mathbf{r}(t)$ of an individual water molecule at time $t$ cannot be evaluated according to deterministic dynamics and is considered as random. It is, therefore, impossible to predict the behavior of an individual water molecule, and the averaged characteristics of the whole ensemble become of interest. For instance, the mean displacement $\mathbb{E}\{[\mathbf{r}(t)-\mathbf{r}(0)]\}$ characterizes coherent motion of water molecules (flow), whereas the mean-square displacement $\mathbb{E}\left\{[\mathbf{r}(t)-\mathbf{r}(0)]^{2}\right\}$ describes fluctuations (throughout the article, the averages or expectations are denoted by $\mathbb{E}$ ). If there is no flow, the mean-square displacement for unrestricted normal diffusion grows linearly with time:

$$
\mathbb{E}\left\{[\mathbf{r}(t)-\mathbf{r}(0)]^{2}\right\}=6 D_{0} t
$$


where $D_{0}$ is the free diffusion coefficient of water molecules.

A complete description of the macroscopic translational dynamics is given by a propagator $G_{t}\left(\mathbf{r}_{0}, \mathbf{r}\right)$, which says how likely it is for a water molecule started from $\mathbf{r}_{0}$ at time 0 to be at $\mathbf{r}$ at a later time $t$. Once the propagator is known, the mean-square displacement and the other averaged characteristics of diffusion can be deduced.

\section{Dynamic ADC}

The internal geometry of a medium, the presence of impenetrable walls or obstacles, as well as trapping, caging, crawding, or other hindering effects may considerably alter or even fully destroy the diffusive character of the macroscopic dynamics. The meansquare displacement either remains linear in time (but with another diffusion coefficient), or exhibit more complex time dependences [(known as anomalous diffusion (3-5)]. In both cases, one can formally introduce an effective, time-dependent or, equivalently, apparent diffusion coefficient (ADC) as

$$
D_{\text {dyn }}(t)=\frac{\mathbb{E}\left\{[\mathbf{r}(t)-\mathbf{r}(0)]^{2}\right\}}{6 t} .
$$

This is a measure how diffusion of nuclei is slowed down by hindering effects. This characteristics, which I call a dynamic ADC, was thoroughly investigated [(see reviews $(2,6,7)$ and references therein)]. Since geometrical compartments (e.g., neurons in the brain) hinder the motion of molecules, ADC contains implicit information, or a "finger-print," of the geometry. Measuring $D_{\text {dyn }}(t)$ is an experimental way to probe biological and mineral porous structures.

\section{Pulsed-Gradient Spin-Echo Technique}

Such measurements can be realized by a pulsed-gradient spin-echo (PGSE) technique (8). After the first $90^{\circ}$ radio-frequency (rf) pulse, the application of a spatially inhomogeneous magnetic field induces phase shifts of the spins that depend on the individual trajectories of the spin-bearing particles. The second $180^{\circ}$ rf pulse partially refocuses the spins that causes the attenuation of the spin-echo amplitude which can be attributed to diffusion. For a free (unrestricted) diffusion in a constant magnetic field gradient of intensity $g$, Hahn and later Carr and Purcell derived the exact formula for the macroscopic signal $E$ at the spin-echo time $t(9,10)$

$$
E=E_{0} \exp \left[-D_{0} \gamma^{2} g^{2} t^{3} / 12\right]
$$

where $\gamma$ is the nuclear gyromagnetic (or magnetogyric) ratio, and $E_{0}$ is the reference signal (without gradient, $g=0$ ). Stejskal and Tanner extended this formula for an arbitrary time-dependent magnetic field gradient $g f(t)(11)$

$$
E=E_{0} \exp \left[-D_{0} \gamma^{2} g^{2} t^{3}\left\langle\left(t_{1}-t_{2}\right)\right\rangle_{2}\right]
$$

where the notation $\left\langle\left(t_{1}-t_{2}\right)\right\rangle_{2}$ from Ref. 2 is for the dimensionless coefficient depending only on the effective dimensionless temporal profile (or gradient waveform) $f(t)$ of the applied magnetic field:

$$
\left\langle\left(t_{1}-t_{2}\right)\right\rangle_{2}=\frac{1}{t^{3}} \int_{0}^{t} d t^{\prime}\left(\int_{0}^{t^{\prime}} d t^{\prime \prime} f\left(t^{\prime \prime}\right)\right)^{2} .
$$

For example, $\left\langle\left(t_{1}-t_{2}\right)\right\rangle_{2}=1 / 12$ for a constant gradient in Eq. [3].

\section{Restricted Diffusion}

When diffusion is restricted, Eqs. [3] and [4] are formally not applicable. The two main "strategies" were proposed to study restricted diffusion.

The first strategy, which can be referred to as "narrow-pulse approximation", was proposed by Tanner and Stejskal (12). A constant gradient is replaced by two gradient pulses of short duration $\delta$. If the nuclei can be considered as immobile during each gradient pulse, the macroscopic signal turns out to be a Fourier transform of the averaged propagator $P(\mathbf{r}, t)$

$$
\begin{aligned}
E & =\int d \mathbf{r} e^{2 \pi i \mathbf{k} \cdot r} P(\mathbf{r}, t), \\
P(\mathbf{r}, t) & =\int d \mathbf{r}_{0} G_{t}\left(\mathbf{r}_{0}, \mathbf{r}_{0}+\mathbf{r}\right),
\end{aligned}
$$

where $\mathbf{k}=\gamma \delta \mathbf{g} /(2 \pi)(8)$. Measuring the signal as a function of $\mathbf{k}$ (and $t$ ) and inverting the Fourier transform, one can access experimentally the averaged propagator of restricted diffusion. An apparent diffusion coefficient can be deduced from the averaged propagator, but the latter contains much richer information about diffusion and a confining medium. A direct access of the averaged propagator made the short time PGSE technique a broadly used tool for studying porous media [see reviews (13-15)]. Although this experimental technique is not in the focus of the article, one should keep in mind this research modaility as an alternative to ADC. 
The second strategy which can be referred to as "Gaussian phase approximation" (GPA) consists in exploring physical situations when Eqs. [3] and [4] either are approximated valid, or can be extended as follows. Measuring the spin-echo signal from water molecules in silica suspensions and sandstones, Woessner observed that formula (Eq. [3]) can still be applied, if $D_{0}$ was replaced by a reduced diffusion coefficient $D$, which he called "a spin-echo diffusion coefficient" (16):

$$
D(t, g)=\frac{-\ln E / E_{0}}{\gamma^{2} g^{2} t^{3}\left\langle\left(t_{1}-t_{2}\right)\right\rangle_{2}} .
$$

The denominator of this formula is often called the $b$-value or $b$-coefficient, allowing one to write the signal in a particularly simple form

$$
\frac{E}{E_{0}}=\exp [-b D]
$$

The relative simplicity and non-invasive character of PGSE measurements made them an attractive and efficient tool for characterizing porous media $(17,18)$. This tool is largely used in various application fields, from lung or brain diffusion-weighted imaging in medicine $(19,20)$ to sedimentary rock analysis in oilrecovery industry $(21,22)$. The apparent diffusion coefficient became a common characteristic of porous media.

In the most general sense, $\mathrm{ADC}$ is a convenient representation of the macroscopic signal through Eq. [6]. As such, it can always be used as a form of contrast enhancement in diffusion-weighted imaging for distinguishing regions with low and high diffusivity. However, a physical interpretation of the spin-echo ADC as a measure of fluctuations can only be recovered in the limit of the gradient strength $g$ going to $0: D_{\lim }(t)=$ $D(t, g=0)$. The limiting value $D_{\lim }(t)$ can be expressed through the second moment of the net dephasing $\varphi$ of the diffusing spins (see Ref. 2 for details)

$$
D_{\lim }(t)=\frac{\mathbb{E}\left\{\varphi^{2} / 2\right\}}{t^{3}\left\langle\left(t_{1}-t_{2}\right)\right\rangle_{2}},
$$

where $\varphi$ is defined here to be dimensional (in units $\mathrm{m} \mathrm{s}$ )

$$
\varphi=\int_{0}^{t} d t^{\prime} f\left(t^{\prime}\right) x\left(t^{\prime}\right)
$$

$x(t)$ being the projection of the random trajectory $\mathbf{r}(t)$ of the spin-bearing particle onto a fixed gradient direction.
For weak gradients (small $g$ ), $D(t, g)$ is close to its limit $D_{\text {lim }}(t)$, so that the signal can be written as

$$
\frac{E}{E_{0}} \simeq \exp \left[-\gamma^{2} g^{2} \mathbb{E}\left\{\varphi^{2} / 2\right\}\right]
$$

This relation, often referred to as "Gaussian-phase approximation," is exact only for free (unrestricted) diffusion, for which all $D_{\mathrm{dyn}}(t), D(t, g)$, and $D_{\mathrm{lim}}(t)$ are time-independent and equal to the free diffusion coefficient $D_{0}$.

The three aforementionned definitions (Eqs. [2], [6], and [8]) are largely used in the literature under the common name of effective, time-dependent, or apparent diffusion coefficient. In what follows, I illustrate that these definitions are not equivalent whenever diffusion is restricted. Although this is perhaps a known fact in a theoretical community [(e.g., see (23)], abundant use and often misuse of apparent diffusion coefficients are still persistent, yielding potential ambiguities in the interpretation of diffusion-weighted experiments. In what follows, I address several fundamental questions about ADCs and its practical use for characterization of porous media.

\section{DOES ONE REALLY MEASURE MEAN- SQUARE DISPLACEMENTS?}

All three ADCs characterize a complex medium through the diffusive motion of spin-bearing particles. The dynamic ADC is a kind of intrinsic characteristic, independent of the applied magnetic field. For this very same reason, $D_{\text {dyn }}(t)$ cannot be measured in a PGSE experiment, though it is still accessible by numerical simulations and theoretical analysis [see $(6,7,24,25)$ and references therein]. By contrast, the spin-echo diffusion coefficient $D(t, g)$ is directly related to experimentally measurable signal, though its physical interpretation is in general less clear. The latter ADC unavoidably depends on the magnetic field used (see, e.g., the explicit dependence on the temporal profile in Eq. [6]). A number of works are devoted to numerical computations of the spin-echo ADC by using Monte Carlo simulations, finite-difference, or finite-element methods (26-32) [for further references, see review (2)].

The above simple but fundamental distinction between the dynamic and spin-echo ADCs indicates that they are not equivalent. This distinction becomes more pronounced in the long-time limit. In a bounded domain, the mean-square displacement in the numerator of Eq. [2] approaches a constant so 
that $D_{\text {dyn }}(t) \propto 1 / t$. In contrast, the second moment $\mathbb{E}\left\{\varphi^{2} / 2\right\}$ is known to behave as $t$, implying $D(t, g) \simeq$ $D_{\lim }(t) \propto 1 / t^{2}(2,23,33)$. The spin-echo diffusion coefficient, therefore, gets smaller and smaller in comparison to the dynamic ADC in the course of time.

Strictly speaking, the dynamic and spin-echo ADCs become equivalent only in the special case of the narrow pulse approximation, when the gradient pulse duration $\delta$ vanishes. However, the NPA is not always appropriate in practice, especially for gases when the spins diffuse fast enough so that the hypothesis of immobile spins during gradient pulses is not applicable.

The only fact that the spin-echo diffusion coefficient is not an intrinsic characteristic of a medium requires much caution for its interpretation. So, two experimental measurements (e.g., with distinct gradient intensity, temporal profile, or timing) may lead to different $D$ in the same sample. This means that reporting ADCs without an accurate specification of the experimental setup is, strictly speaking, meaningless. Unfortunately, it became a common practice, especially in the medical NMR literature, to characterize quantitatively a complex medium through an ADC by using a single $b$-value instead of giving the complete set of physical parameters. This oversimplification is exaggarated. Although the $b$-value is a useful characteristic, it is not representative of the whole experimental setup.

\section{IS ADC ALWAYS MEANINGFUL?}

Another important issue for restricted diffusion is the difference between the spin-echo diffusion coefficient $D(t, g)$ computed directly from the signal in a PGSE experiment, and its counterpart $D_{\lim }(t)$, which is extrapolated from data in the limit of $g$ going to 0 . According to Eq. [6], the former ADC is not only a function of time, but also a function of the gradient intensity $g$. In Woessner's experiment, as well as in many others, the dependence of ADC on $g$ was not observed, i.e., $\ln E$ was proportional to $\gamma^{2} g^{2}$. This empirical observation can be related to Gaussian phase approximation which, depending on experimental conditions, may be valid or not.

Limitations of the Gaussian phase approximation and the underlying $g^{2}$ dependence (Eq. [9]) are known for a long time. Using narrow gradient pulses, Callaghan et al. discovered the emergence of diffusion-diffraction patterns, which evidently contradict the GPA (34). Balinov and coworkers observed diffraction-like effects in the PGSE experiment when applied to a highly concentrated water-oil emulsion (35). Kuchel et al. presented data showing the diffusion-diffraction effect of water in cell suspensions and the ability to determine cell dimension(s) from these data (36). In all these cases, the macroscopic signal as a function of gradient intensity is used to characterize restricted diffusion and a porous medium, without appealing to ADC [see reviews (1315) for further details].

A breakdown of the GPA in a constant gradient was predicted by Stoller and coworkers for restricted diffusion in a slab (37) and further discussed by de Swiet and Sen (38). The related non-Gaussian stretched-exponential behavior was then experimentally observed by Hürlimann et al. for restricted diffusion of water molecules between two parallel plates (39). A drastic deviation from the Gaussian $g^{2}$-dependence of $\ln E$ was observed at gradient intensities above $15 \mathrm{mT} \mathrm{m}^{-1}$. As a consequence, the spin-echo diffusion coefficient from Eq. [6] strongly depended on $g$, losing its physical interpretation as a "diffusion coefficient." In other words, $D(t, g)$ was not a better characterization of the macroscopic dynamics than the signal $E$ itself. It is worth stressing that the experimental conditions in Hürlimann's experiment were ordinary. Much larger gradients are now accessible on modern MR scanners so that a non-Gaussian signal attenuation may be relevant for clinical applications (40-46). For instance, Jacob and coworkers demonstrated a clear deviation from a linear dependence of $\ln E / E_{0}$ on $b$ for helium-3 gas diffusion in the rat lung (44).

Although $D(t, g)$ may depend on $g$, its limiting value $D(t)$ is related to the second moment of the total dephasing which is independent of the gradient intensity $g$. When the GPA is applicable, the second moment mostly determines the signal according to Eq. [9], and $D_{\lim }(t)$ and $D(t)$ are close to each other. When the GPA does not hold, $D_{\lim }(t)$ is still well defined (as the second moment itself), but it cannot be directly accessed by a PGSE technique.

To illustrate the above arguments, I calculated ADCs for restricted diffusion in a slab under the conditions similar to those of the experiment by Hürlimann et al. (39). The calculation was based on the multiple correlation function technique explained in detail in $(2,47)$. This technique relies on the Laplace operator eigenbasis which is explicitly known for a slab. The physical parameters used are: the slab width $L$ is $0.16 \mathrm{~mm}$, the gyromagnetic (or magnetogyric) ratio $\gamma$ for protons is $2.675 \times 10^{8} \mathrm{rad} \mathrm{T}^{-1} \mathrm{~s}^{-1}$, and the pulse duration $\delta$ is the half of the echo time $t$ (the case of a constant gradient). The echo time $t$ and the gradient intensity $g$ can be varied in relatively 
broad ranges, usually taking the values between 1 and $100 \mathrm{~ms}$ for $t$, and between 1 and $100 \mathrm{mT} \mathrm{m}^{-1}$ for $g$. For our illustrative purposes, it is convenient to fix these parameters and vary the free diffusion coefficient $D_{0}$ instead. If one takes $t=100 \mathrm{~ms}$, the dimensionless ratio $D_{0} t / L^{2}$ can be varied from 0.01 (with $D_{0} \simeq 2.3 \times 10^{-9} \mathrm{~m}^{2} \mathrm{~s}^{-1}$ for water molecules under ambient conditions) to several hundred for gases (e.g., $D_{0} \simeq 2 \times 10^{-4} \mathrm{~m}^{2} \mathrm{~s}^{-1}$ for helium-3 under ambient conditions). Note that large free diffusion coefficients made gases particularly appropriate for probing porous media $(48,49)$.

Figure 1 shows various ADCs as functions of the dimensionless ratio $D_{0} t / L^{2}$ for two values of the gradient intensity: $g=1 \mathrm{mT} \mathrm{m}^{-1}$ and $g=15 \mathrm{mT} \mathrm{m}^{-1}$. In the former case, the Gaussian phase approximation holds, and the spin-echo diffusion coefficient $D(t, g)$ is close to its limit $D_{\lim }(t)$ (at $g=0$ ) determined by the second moment. The situation is different for a larger gradient $g=15 \mathrm{mT} \mathrm{m}^{-1}$, for which the dependences $D(t, g)$ and $D_{\lim }(t)$ on time are distinct. In this case, the GPA does not hold making the estimate of the spinecho diffusion coefficient derived from a PGSE experiment unrelated to the real intrinsic value.

Figure 2 shows the difference between dynamic and spin-echo ADCs. In the slow diffusion or shorttime limit $\left(D_{0} t / L^{2}<0.1\right)$, the spin-echo ADC with $g=1 \mathrm{mT} \mathrm{m}^{-1}$ and its limit $D_{\lim }(t)$ reproduce the dependence $D_{\text {dyn }}(t)$ with accuracy better than $10 \%$.

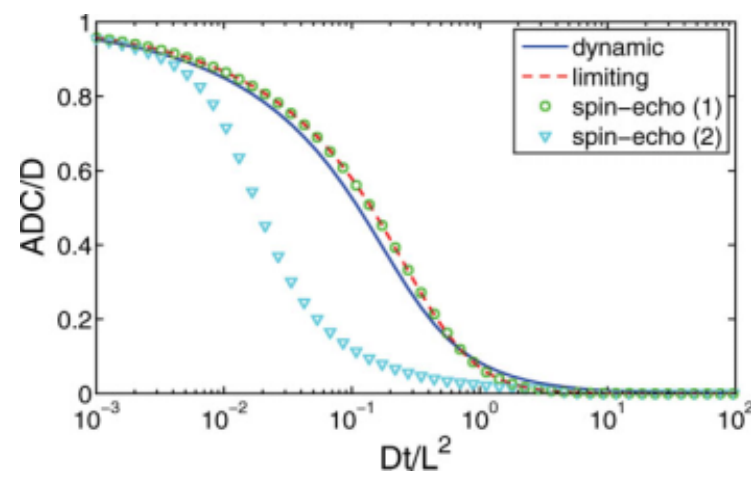

Figure 1 Various ADCs for restricted diffusion in a slab: $\mathrm{D}_{\mathrm{dyn}}(t)$ (solid line), $\mathrm{D}_{\text {lim }}(t)$ (dashed line), $D(t, g)$ for $g=1 \mathrm{mT} \mathrm{m}^{-1}$ (circles), $D(t, g)$ for $g=15 \mathrm{mT} \mathrm{m}^{-1}$ (triangles). When $g=1 \mathrm{mT} \mathrm{m}^{-1}$, the spin-echo ADC $D(t, g)$ and its limit $D_{\text {lim }}(t)$ are close to each other. In contrast, for $g=15 \mathrm{mT} \mathrm{m}^{-1}$, the Gaussian phase approximation breaks down, and the estimated spin-echo diffusion coefficient, though still defined, is substantially different from the true intrinsic value. Note that the experiment by Hürlimann and coworkers corresponded to $D_{0} t / L^{2} \simeq 0.009$ (39). [Color figure can be viewed in the online issue, which is available at www.interscience.wiley.com.]

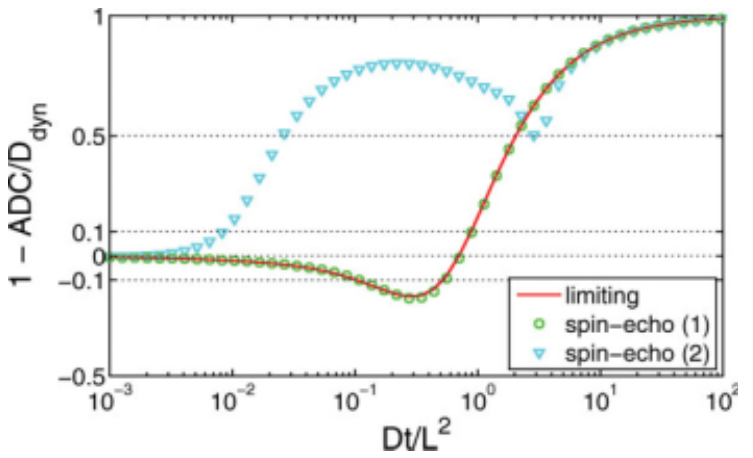

Figure 2 Relative error between $D_{\text {dyn }}(t)$ and three other ADCs from Fig. 1. When $D_{0} t / L^{2}$ is less than 0.1 , the spinecho ADC with $g=1 \mathrm{mT} \mathrm{m}^{-1}$ and its limit $D_{\text {lim }}(t)$ reproduce $D_{\text {dyn }}(t)$ with error less than $10 \%$. The difference becomes increasingly sharper for larger values of $D_{0} t / L^{2}$. When $D_{0} t / L^{2}$ exceeds 10 , all three spin-echo ADCs (behaving as $1 / t^{2}$ ) are small in comparison to $D_{\mathrm{dyn}}(t)$ (behaving as $1 / t$ ). [Color figure can be viewed in the online issue, which is available at www.interscience.wiley.com.]

In the long-time limit $\left(D_{0} t / L^{2}>10\right)$, all three spinecho ADCs are small in comparison to $D_{\mathrm{dyn}}(t)$, and the relative error approaches 1 . This is in agreement with the aforementioned theoretical behaviors $D_{\mathrm{dyn}}(t)$ $\propto 1 / t$ and $D(t, g) \simeq D_{\lim }(t) \propto 1 / t^{2}$. It is worth stressing that the long-time limit of restricted diffusion is considered in a bounded domain. Although the asymptotic behavior of ADCs for unbounded porous media is different (7), the distinction between dynamic and spin-echo ADCs is still expected.

The transition to a non-Gaussian regime is partly investigated in $(2,37,38,50,51)$. In simple confinements such as a slab, the Gaussian phase approximation is known to break down when the dephasing length $\left(D_{0} / \gamma g\right)^{1 / 3}$ is much smaller than the diffusion length $\sqrt{D_{0} t}$ and the characteristic dimension $L$ of the domain. For instance, in the experiment by Hürlimann et al.: $L=160 \mu \mathrm{m}, \sqrt{D_{0} t} \simeq 15 \mu \mathrm{m}$, and $\left(D_{0} /\right.$ $\gamma g)^{1 / 3}$ was $\sim 20 \mu \mathrm{m}$ for $g=1 \mathrm{mT} \mathrm{m}^{-1}$ and $8 \mu \mathrm{m}$ for $g=15 \mathrm{mT} \mathrm{m}^{-1}$. In the latter case, the distinction between $D(t, g)$ and $D_{\lim }(t)$ was already significant (see Fig. 1 for $D_{0} t / L^{2} \simeq 0.009$ ), and it still increased for larger $D_{0}$. In general, the smallness of the kurtosis, $\mathbb{E}\left\{\varphi^{4}\right\} /\left[\mathbb{E}\left\{\varphi^{2}\right\}\right]^{2}-3$, is the first condition to check for the applicability of the GPA. Further theoretical and numerical studies of this problem for porous media are important.

\section{WHICH FACTORS DO INFLUENCE ADC?}

In the previous analysis, I have focused on the main differences between the dynamic and spin-echo 
ADCs, the latter being dependent on, and thus, sensitive to particular experimental conditions. Here, I briefly comment on several factors which can substantially modify the spin-echo ADC.

\section{Temporal Profile $f(t)$ of the Magnetic Field}

The temporal profile can be largely varied in experiments. Usually, one uses a bipolar gradient with two trapezoidal (or rectangular) pulses. For the same time delay $t$ between the first rf pulse and the echo, the duration and shape of the gradient pulses can be different, and this variation affects the spin-echo ADC. As an example, I consider two rectangular pulses of duration $\delta$, for which the integral in Eq. [5] becomes $t^{3}\left\langle\left(t_{1}-t_{2}\right)\right\rangle_{2}=t \delta^{2}-4 \delta^{3} / 3$. In the long-time regime, the asymptotic behavior of the second moment implies the following dependence on $t$ and dimensionless ratio $\delta / t(2)$

$$
D_{\lim }(t) \propto \frac{2}{(\delta / t)[1-(\delta / t) 4 / 3]} t^{-2} .
$$

The case $\delta=t / 2$ describes a constant gradient. By varying only the duration $\delta$, the ADC can be changed by orders of magnitude. In other words, two measurements, which differ only by temporal profiles, would give different ADCs for the same sample. Although I considered here the long-time regime for illustrative purposes, the dependence on the temporal profile remains relevant for all diffusion times.

\section{Spatial Profile of the Magnetic Field}

In most PGSE experiments on restricted diffusion, a linear magnetic field gradient is applied. However, the difference in magnetic susceptibility between two phases (e.g., air/tissue phases in the lungs or water/ solid phases in mineral samples such as sedimentary rocks) may raise local internal magnetic fields near the interface between two phases. The signal attenuation and the consequent value of ADC are determined by an effective gradient which is the sum of an applied (and well-controlled) external gradient and an internal gradient whose spatial distribution is in general unknown and difficult to take into account in experiment.

At the same time, the effect of various spatial profiles of the magnetic field can be studied theoretically or numerically (51-55). For instance, the cosine spatial profile was considered as a simplified model for susceptibility-induced magnetic fields in a slab geometry (55). For this specific case, the second moment was found exactly in Ref. 51. For two rectangular gradient pulses of duration $\delta=t / 2$, it was found that

$$
D_{\lim }^{\cos }(t)=\frac{12}{p \pi^{2}}\left(\frac{1}{p \pi^{2}}-\frac{e^{-p \pi^{2}}-4 e^{-p \pi^{2} / 2}+3}{\left(p \pi^{2}\right)^{2}}\right)
$$

where $p=D_{0} t / L^{2}$. This dependence is close to, but not identical with, $D_{\lim }(t)$ and $D(t)$ for a linear gradient.

Note that the presence of internal gradients may be advantageous. For instance, a stronger attenuation of the signal near the frontier yields an edge enhancement $(56,57)$. Internal gradients can also be used to obtain pore size distribution of porous media $(22,58)$.

\section{Surface Relaxation}

Surface relaxation can also modify the spin-echo ADC in a complex manner. In sharp contrast with bulk relaxation which acts uniformly over the sample and can, thus, be factored out, surface relaxation affects the statistics of Brownian trajectories, eliminating those which encounter the boundary more frequently. These effects were intensively studied [e.g., (50, 59-62); see also reviews $(2,7)$ and references therein].

\section{Surface Permeability}

For biological applications, the walls of a geometrical compartment are often semi-permeable so that water molecules can leave a studied compartment (e.g., a living cell) or travel between compartments. These exchanges make the analysis of restricted diffusion and a reliable interpretation of experimental data more difficult. The role of semi-permeable boundaries was thoroughly investigated $(2,63-66)$ so that these issues are not addressed to in the present article. One can mention the work by Kuchel and Durrant who considered models with unevenly spaced semi-permable parallel membranes (67), and the work by Sen who suggested to use time-dependent diffusion coefficient as a probe of permeability of the pore wall (68). A model for diffusive transport through a spherical interface probed by pulsed-gradient spin-echo technique was developed in $(69,70)$.

\section{CAN ONE GO BEYOND ADC?}

If the Gaussian phase approximation and the underlying $g^{2}$ dependence (Eq. [9]) is inconsistent for a 
studied system, the computation of apparent diffusion coefficient is of limited use. Nonetheless, one can still extract useful information about a confining medium from the macroscopic signal as a function of gradient intensity or echo time. How can one fit data? Let me distinguish several "strategies" that may help to answer this question.

1. When the duration of gradient pulses is very short, the macroscopic signal can be seen as a Fourier transform of the averaged propagator [see reviews (13-15) and references therein]. In particular, the emergence of diffusion-diffraction patterns allows one to determine a typical length scale of the medium $(28,34$ 36). This is a powerful experimental tool which is broadly used for characterization of porous media in material sciences. This strategy is applicable when nuclei diffuse slowly enough to be considered as immobile during the gradient pulses. This is often the case for liquids and sometimes for gases.

2. For simple confining domains (such as slab, cylinder, sphere, cylindrical, and spherical shells), the computation of the macroscopic signal by using matrix formalisms is easy, rapid, and very accurate $(2,47,50,62,71-$ 76). Unknown parameters (such as the compartment size or free diffusion coefficient) can be extracted by fitting experimental data to numerical curves. This strategy is limited to simple confining domains, whose shapes are supposed to be known a priori.

3. For complex domains, the macroscopic signal can be found numerically (by Monte Carlo simulations, finite difference, or finite element methods, etc.) if their shape is known a priori. For instance, one can compute the signal from the nuclei diffusing in a pack of spherical beads. This strategy is useful for calibrating experimental techniques in test samples.

4. When the geometry of a confining medium is not known, one often attempts to use model formulas for fitting experimental data. The adjustable parameters which are determined from fitting, serve for characterization of the geometrical structure. In what follows, I briefly discuss several model formulas which are broadly used for fitting the macroscopic signal.

\section{Bi-Exponential Model}

In some cases, two separate "compartments" of a confining medium can be naturally distinguished (e.g., intracellular and extracellular spaces). If diffusion of nuclei within each compartment can be modeled as unrestricted, the macroscopic signal is the sum of monoexponential contributions (Eq. [7]) from each compartment:

$$
\frac{E}{E_{0}}=\zeta e^{-b D_{\mathrm{S}}}+(1-\zeta) e^{-b D_{\mathrm{F}}}
$$

where $D_{\mathrm{S}}$ and $D_{\mathrm{F}}$ denote the apparent diffusion coefficients in each compartment (subscripts $\mathrm{S}$ and $\mathrm{F}$ conventionally refer to "slow" and "fast" motion), and $\zeta$ and $(1-\zeta)$ are the relative volume fractions of these compartments. This bi-exponential model is often used to interpret the signal attenuation in biological systems and porous media (77-81).

Despite numerous successful applications, Eq. [10] is a fitting formula which may be valid or not. I highlight two limiting factors.

- When the structure of a studied confining medium is expected to have separate compartments, the presence of a separating interface implies restricted diffusion for which Gaussian contributions in Eq. [10] are approximate. Although the use of a bi-exponential model is justified from a geometrical point of view, one needs to check that the Gaussian phase approximation is applicable for each compartment. This is often the case for small $b$-values.

- When there is no a priori evidence that a confining medium has separate compartments, the use of a bi-exponential model for fitting experimental data may be misleading: a good fit by Eq. [10] does not necessarily signify the existence of two separate compartments with different diffusion coefficients. This point was recently illustrated by Milne and Conradi who observed a bi-exponential signal decay from diffusion in a single (cylindrical) compartment (82).

I go a step further and use the experiment by Milne and Conradi to illustrate another "danger" of fitting models. To reproduce their results, I calculate the macroscopic signal for the physical parameters used in (82): $r=160 \mu \mathrm{m}$ (radius of cylinders), $\delta=$ $3 \mathrm{~ms}$ (duration of gradient pulses), $\Delta=138 \mathrm{~ms}$ (echo time), $D_{0}=0.00173 \mathrm{~mm}^{2} \mathrm{~s}^{-1}$ (free diffusion coefficient of water molecules), and $b$-value ranging from 0.15 to $6,000 \mathrm{~s} \mathrm{~mm}^{-2}$. The macroscopic signal and its bi-exponential fit are shown in Fig. 3. If one restricts itself for the range of the $b$-values used in (82), an excellent fit may suggest that a 


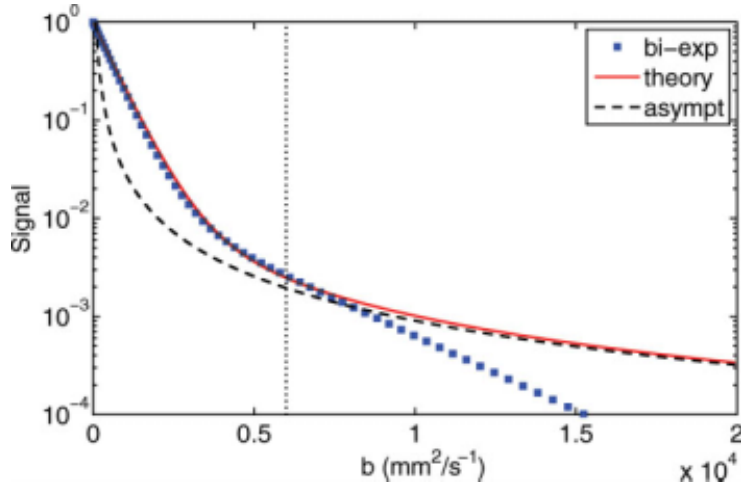

Figure 3 Normalized signal for the cylinder of radius $r=$ $160 \mu \mathrm{m}$ with diffusion gradient perpendicular to the long axis. The bi-exponential fit (Eq. [10]) (shown by squares) is compared to a numerical solution (solid line) and powerlaw asymptotic behavior (11) (dashed line). A vertical dotted line delimits the region on the left $\left(b \leq 6,000 \mathrm{~s} \mathrm{~mm}^{-2}\right)$, which was considered in (82). In this region, the bi-exponential fit reproduces accurately theoretical predictions. A deviation from this "effective" regime is clear on the right. [Color figure can be viewed in the online issue, which is available at www.interscience.wiley.com.]

bi-exponential model really describes the signal attenuation due to restricted diffusion in cylinders. This conclusion, which seems to rely on the experimental evidence, is false. A closed analytical formula for the signal from a cylindrical confinement for narrow gradient pulses is well known $(83,84)$. Moreover, Debye-Porod power-law decay of the signal in the slow-diffusion regime at large $g$ (or $b$ ) was demonstrated $(85,86)$ :

$$
E \propto g^{-3} \propto b^{-3 / 2} \quad(b \gg 1 / D) .
$$

This power-law decay is in sharp contrast to the exponential decay suggested by Eq. [10].

Figure 3 clearly shows a deviation from a bi-exponential fit for $b$-values larger than $6,000 \mathrm{~s} \mathrm{~mm}^{-2}$. This means that for cylinders, the bi-exponential formula is nothing more than an approximation for a limited range of $b$-values. An interpretation of the adjustable parameters $D_{\mathrm{S}}, D_{\mathrm{F}}$, and $\zeta$ of the bi-exponential model would be superficial. Moreover, the values of these parameters strongly depend on the range of $b$-values that are used for fitting. Other limitations of a bi-exponential model are discussed in (45).

\section{Distributed-Exponential Model}

Porous media and biological tissues often exhibit multiple lengths scales. Assuming that each compartment is characterized by its own apparent diffusion coefficient $D$, the macroscopic signal from the whole medium can be approximated as a collection of uncoupled exponential decay processes:

$$
\frac{E}{E_{0}}=\int_{0}^{\infty} d D e^{-b D} p(D),
$$

where $p(D)$ is a distribution of apparent diffusion coefficients. Once the macroscopic signal $E$ as a function of the $b$-value is measured, this integral relation can be inverted to obtain the $p(D)$. This distribution tells us about relative fractions of different compartments of a confining medium. However, this model is rarely used in practice because a numerical inversion of the Laplace transform requires accurate measurements of the macroscopic signal for a large range of $b$-values.

\section{Stretched-Exponential Model}

A broad distribution of apparent diffusion coefficients is often represented by a stretched-exponential model

$$
\frac{E}{E_{0}}=\exp \left[-(b D)^{\alpha}\right]
$$

where $\alpha$ is the stretching parameter, which characterizes the deviation of the signal from Gaussian (monoexponential) behavior. Higher heterogeneity of a confining medium typically corresponds to smaller values of $\alpha$, the latter serving thus as a geometrical characteristic of the medium. A stretched-exponential model was proposed to characterize continuously distributed cortical water diffusion rates (87), whereas its modification was used for the characterization of transverse and longitudinal NMR relaxation of fluid in saturated rock cores (88). One of the limitations of the stretched-exponential model is that the relation between the stretching parameter $\alpha$ and the geometrical structure of a confining medium remains obscure.

\section{Kurtosis and Higher Moments}

Another extension of the classical formula (Eq. [7]) is based on the cumulant expansion for the macroscopic signal [see (2) for details]. The kurtosis fitting formula is obtained when the cumulant expansion is truncated to the second-order term:

$$
\frac{E}{E_{0}}=\exp \left[-b D+b^{2} K D^{2} / 6\right]
$$

where the parameter $K$ is called the (apparent) diffusion kurtosis. The smaller the kurtosis, the closer the 
distribution of the phase shifts is to the Gaussian one. Since heterogeneity of a confining domain is responsible for the deviation from the Gaussian behavior (typical for homogeneous media), kurtosis imaging was suggested to characterize the tissue structure (41, 43). One limitation of the model formula (Eq. [14]) is an unlimited growth of the macroscopic signal with $b$. Still higher-order moments have to be included to correct this unphysical increase of the signal. In practical terms, it means that the kurtosis fitting formula can only be applied for a limited range of the $b$-values.

\section{CONCLUSION}

I revisited the subtle but fundamental differences between the dynamic diffusion coefficient $D_{\mathrm{dyn}}(t)$ quantifying the mean-square displacement of diffusing particles, and its spin-echo counterpart $D(t)$ measured by a pulsed-gradient spin-echo technique. Although both ADCs allow one to characterize restricted diffusion and porous media, the former is an intrinsic characteristic, whereas the latter unavoidably depends on the experimental setup. As a consequence, the information extracted from the spin-echo ADC cannot be considered independently of measurement conditions. Unless ADC is used as a form of contrast enhancement, it is important that reported ADC values be accompanied by an accurate specification of the experimental setup (gradient intensity and waveform, timing, etc.). The use of a single $b$ value is oversimplified for a quantitative analysis. For weak gradient and slow diffusion, the spin-echo ADC is a good approximation to the dynamic one. For larger gradients, the measureable quantity $D$ may depend on the gradient intensity, losing its proper physical interpretation as a diffusion coefficient. In a non-Gaussian regime, the use of the spin-echo ADC as a characteristic of a porous medium is not justified and may be strongly misleading. In particular, the extraction of an ADC from two measurements at $g=$ 0 (reference signal) and $g \neq 0$ (attenuated signal) should be avoided if an accurate value of ADC is needed. At least one another measurement is required to check that the Gaussian phase approximation is valid and the estimated ADC is independent of the gradient intensity. Finding a general reliable criterion for the applicability of the GPA in porous media is an important issue.

A violation of the Gaussian phase approximation is a consequence of a geometrical restriction. Any deviation from the classical formula (Eq. [7]) is a potentially interesting characteristic of complex porous media. To extract such characteristics, several models were proposed for fitting experimental data of diffusion-weighted imaging. Their adjustable parameters, such as fast and slow ADCs $D_{\mathrm{F}}$ and $D_{\mathrm{S}}$, stretching parameter $\alpha$, kurtosis $K$, etc., are sensitive to heterogeneity of a confining domain. As a result, a failure of the GPA may bring finer information about porous media and biological tissues than a classical measurement of apparent diffusion coefficients.

\section{ACKNOWLEDGMENTS}

This work has been supported by the ANR project "DYOPTRI." The author thank Dr. G. Guillot, Dr. P. Levitz, and the referee for their helpful comments.

\section{REFERENCES}

1. Klafter J, Drake JM. 1989. Molecular Dynamics in Restricted Geometries. New York: John Wiley and Sons.

2. Grebenkov DS. 2007. NMR survey of reflected brownian motion. Rev Mod Phys 79:1077-1137.

3. Bouchaud JP, Georges A. 1990. Anomalous diffusion in disordered media: statistical mechanisms, models and physical applications. Phys Rep 195:127-293.

4. Metzler R, Klafter J. 2000. The random walk's guide to anomalous diffusion: a fractional dynamics approach. Phys Rep 339:1-77.

5. Kimmich R. 2002. Strange kinetics, porous media, and NMR. Chem Phys 284:253-285.

6. Haus JW, Kehr KW. 1987. Diffusion in regular and disordered lattices. Phys Rep 150:263-406.

7. Sen PN. 2004. Time-dependent diffusion coefficient as a probe of geometry. Conc Magn Reson A 23:121.

8. Callaghan PT. 1991. Principles of Nuclear Magnetic Resonance Microscopy. Oxford: Clarendon Press.

9. Hahn EL. 1950. Spin echoes. Phys Rev 80:580-594.

10. Carr HY, Purcell EM. 1954. Effects of diffusion on free precession in NMR experiments. Phys Rev 94: 630-638.

11. Stejskal EO, Tanner JE. 1965. Spin diffusion measurements: spin echoes in the presence of a timedependent field gradient. J Chem Phys 42:288-292.

12. Tanner JE, Stejskal EO. 1968. Restricted self-diffusion of protons in colloidal systems by the pulsedgradient, spin-echo method. J Chem Phys 49:17681777.

13. Price WS. 1997. Pulsed-field gradient nuclear magnetic resonance as a tool for studying translational diffusion: Part 1. Basic theory. Conc Magn Reson 9: 299-336. 
14. Price WS. 1998. Pulsed-field gradient nuclear magnetic resonance as a tool for studying translational diffusion: Part 2. Experimental aspects. Conc Magn Reson 10:197-237.

15. Callaghan PT, Codd SL, Seymour JD. 1999. Spatial coherence phenomena arising from translational spin motion in gradient spin echo experiments. Conc Magn Reson 11:181-202.

16. Woessner DE. 1963. NMR spin-echo self-diffusion measurements on fluids undergoing restricted diffusion. J Phys Chem 67:1365-1367.

17. Watson AT, Chang CTP. 1997. Characterizing porous media with NMR methods. Prog Nucl Magn Reson Spectrosc 31:343-386.

18. Barrie PJ. 2000. Characterization of porous media using NMR methods. Annu Rep NMR Spectrosc 41: 265-316.

19. van Beek EJR, Wild JM, Kauczor HU, Schreiber W, Mugler JP, de Lange EE. 2004. Functional MRI of the lung using hyperpolarized 3-helium gas. J Magn Reson Imag 20:540-554.

20. Conradi MS, Saam B, Yablonskiy DA, Woods JC. 2006. Hyperpolarized $3 \mathrm{He}$ and perfluorocarbon gas diffusion MRI of lungs. Prog Nucl Magn Reson Spectr 48:63-83.

21. Hürlimann MD, Helmer KG, Latour LL, Sotak CH. 1994. Restricted diffusion in sedimentary rocks. Determination of surface-area-to-volume ratio and surface relaxivity. J Magn Reson A 111:169-178.

22. Song YQ, Ryu S, Sen PN. 2000. Determining multiple length scales in rocks. Nature (London) 406:178181.

23. Helmer KG, Hürlimann MD, de Swiet TM, Sen PN, Sotak CH. 1995. Determination of ratio of surface area to pore volume from restricted diffusion in a constant field gradient. J Magn Reson A 115:257259.

24. Kirkpatrick TR. 1982. Time dependent transport in a fluid with static traps. J Chem Phys 76:4255-4259.

25. de Swiet TM, Sen PN. 1996. Time dependent diffusion coefficient in a disordered medium. J Chem Phys 104:206-209.

26. Balinov B, Jönsson B, Linse P, Söderman O. 1993. The NMR self-diffusion method applied to restricted diffusion. Simulation of echo attenuation from molecules in spheres and between planes. J Magn Reson A 104:17-25.

27. Blees MH. 1994. The effect of finite duration of gradient pulses on the pulsed-field-gradient NMR method for studying restricted diffusion. J Magn Reson A 109:203-209.

28. Lennon AJ, Kuchel PW. 1994. Neural networks used to interpret pulsed-gradient restricted-diffusion data. J Magn Reson A 107:229-235.

29. Coy A, Callaghan PT. 1994. Pulsed gradient spin echo nuclear magnetic resonance for molecules diffusing between partially reflecting rectangular barriers. J Chem Phys 101:4599-4609.
30. Linse P, Söderman O. 1995. The validity of the short-gradient-pulse approximation in NMR studies of restricted diffusion. Simulations of molecules diffusing between planes, in cylinders and spheres. J Magn Reson A 116:77-86.

31. Kuchel PW, Lennon AJ, Durrant C. 1996. Analytical solutions and simulations for spin-echo measurements of diffusion of spins in a sphere with surface and bulk relaxation. J Magn Reson B 112:1-17.

32. Hagslätt H, Jönsson B, Nydén M, Söderman O. 2003. Predictions of pulsed field gradient NMR echo-decays for molecules diffusing in various restrictive geometries. Simulations of diffusion propagators based on a finite element method. J Magn Reson 161:138-147.

33. Robertson B. 1966. Spin-echo decay of spins diffusing in a bounded region. Phys Rev 151:273-277.

34. Callaghan PT, Coy A, MacGowan D, Packer KJ, Zelaya FO. 1991. Diffraction-like effects in NMR diffusion studies of fluids in porous solids. Nature (London) 351:467-469.

35. Balinov B, Söderman O, Ravey J-C. 1994. Diffraction-like effects observed in the PGSE experiment when applied to a highly concentrated water-oil emulsion. J Phys Chem 98:393-395.

36. Kuchel PW, Coy A, Stilbs P. 1997. NMR "diffusiondiffraction" of water revealing alignment of erythrocytes in a magnetic field and their dimensions and membrane transport characteristics. Magn Reson Med 37:637-643.

37. Stoller SD, Happer W, Dyson FJ. 1991. Transverse spin relaxation in inhomogeneous magnetic fields. Phys Rev A 44:7459-7477.

38. de Swiet TM, Sen PN. 1994. Decay of nuclear magnetization by bounded diffusion in a constant field gradient. J Chem Phys 100:5597-5604.

39. Hürlimann MD, Helmer KG, de Swiet TM, Sen PN, Sotak CH. 1995. Spin echoes in a constant gradient and in the presence of simple restriction. J Magn Reson A 113:260-264.

40. Alexander DC, Barker GJ, Arridge SR. 2002. Detection and modeling of non-Gaussian apparent diffusion coefficient profiles in human brain data. Magn Reson Med 48:331-340.

41. Jensen JH, Helpern JA, Ramani A, Lu H, Kaczynski K. 2005. Diffusional Kurtosis imaging: the quantification of non-Gaussian water diffusion by means of magnetic resonance imaging. Magn Reson Med 53:1432-1440.

42. Liu C, Bammer R, Moseley ME. 2005. Limitations of apparent diffusion coefficient-based models in characterizing non-Gaussian diffusion. Magn Reson Med 54:419-428.

43. Trampel R, Jensen JH, Lee RF, Kamenetskiy I, McGuinness G, Johnson G. 2006. Diffusional Kurtosis imaging in the lung using hyperpolarized $3 \mathrm{He}$. Magn Reson Med 56:733-737.

44. Jacob RE, Laicher G, Minard KR. 2007. 3D MRI of non-Gaussian $3 \mathrm{He}$ gas diffusion in the rat lung. J Magn Reson 188:357-366. 
45. Kiselev VG, Il'yasov KA. 2007. Is the "biexponential diffusion" biexponential? Magn Reson Med 57:464469.

46. Grebenkov DS, Guillot G, Sapoval B. 2007. Restricted diffusion in a model acinar labyrinth by NMR. Theoretical and numerical results. J Magn Reson 184:143-156.

47. Grebenkov DS. 2008. Laplacian Eigenfunctions in NMR. I. A numerical Tool. Conc Magn Reson A 32: 277-301.

48. Mair RW, Cory DG, Peled S, Tseng CH, Patz S, Walsworth RL. 1998. Pulsed-field-gradient measurements of time-dependent gas diffusion. J Magn Reson 135:478-486.

49. Mair RW, Wong GP, Hoffmann D, Hürlimann MD, Patz S, Schwartz LM, et al. 1999. Probing porous media with gas diffusion NMR. Phys Rev Lett 83: 3324-3327.

50. Axelrod S, Sen PN. 2001. Nuclear magnetic resonance spin echoes for restricted diffusion in an inhomogeneous field: methods and asymptotic regimes. J Chem Phys 114:6878-6895.

51. Grebenkov DS. 2007. NMR restricted diffusion between parallel planes in a cosine magnetic field: an exactly solvable model. J Chem Phys 126:104706-115.

52. Lisitza NV, Song Y-Q. 2001. The behavior of diffusion eigenmodes in the presence of internal magnetic field in porous media. J Chem Phys 114:9120-9124.

53. Sigmund EE, Cho H, Chen P, Byrnes S, Song Y-Q, Guo XE, et al. 2008. Diffusion-based MR methods for bone structure and evolution. Magn Reson Med 59:28-39.

54. Cho H, Ryu S, Ackerman JL, Song Y-Q. 2009. Visualization of inhomogeneous local magnetic field gradient due to susceptibility contrast. J Magn Reson 198:88-93.

55. Zielinski LJ, Sen PN. 2000. Relaxation of nuclear magnetization in a nonuniform magnetic field gradient and in a restricted geometry. J Magn Reson 147: 95-103.

56. Pütz B, Barsky D, Schulten K. 1992. Edge enhancement by diffusion in microscopic MRI. J Magn Reson 97:27-53.

57. de Swiet TM. 1995. Diffusive edge enhancement in imaging. J Magn Reson B 109:12-18.

58. Song YQ. 2003. Using internal magnetic fields to obtain pore size distributions of porous media. Conc Magn Reson A 18:97-110.

59. Mitra PP, Sen PN. 1992. Effects of microgeometry and surface relaxation on NMR pulsed-field-gradient experiments: simple pore geometries. Phys Rev B 45: 143-156.

60. Grebenkov DS. 2007. Multiple correlation function approach: rigorous results for simple geometries. Diff Fundam 5:1-34.

61. Grebenkov DS. 2008. Analytical solution for restricted diffusion in circular and spherical layers in inhomogeneous magnetic fields. J Chem Phys 128: 134702-1-14.

62. Codd SL, Callaghan PT. 1999. Spin echo analysis of restricted diffusion under generalized gradient waveforms: planar, cylindrical and spherical pores with wall relaxivity. J Magn Reson 137:358-372.

63. Sapoval B. 1994. General formulation of Laplacian transfer across irregular surfaces. Phys Rev Lett 73: 3314-3317.

64. Felici M, Filoche M, Straus C, Similowski T, Sapoval B. 2005. Diffusional screening in real 3D human acini: a theoretical study. Resp Physiol Neurobiol 145:279-293.

65. Grebenkov DS, Filoche M, Sapoval B. 2006. Mathematical basis for a general theory of laplacian transport towards irregular interfaces. Phys Rev E 73: 021103 .

66. Grebenkov DS. 2006. Partially reflected brownian motion: a stochastic approach to transport phenomena. In: Velle LR ed. Focus on Probability Theory. Hauppauge. Nova Science Publishers. pp. 135-169.

67. Kuchel PW, Durrant CJ. 1999. Permeability coefficients from NMR q-space data: models with unevenly spaced semi-permeable parallel membranes. J Magn Reson 139:258-272.

68. Sen PN. 2003. Time-dependent diffusion coefficient as a probe of permeability of the pore wall. J Chem Phys 119:9871-9876.

69. Barzykin AV, Hayamizu K, Price WS, Tachiya M. 1995. Pulsed-field-gradient NMR of diffusive transport through a spherical interface into an external medium containing a relaxation agent. J Magn Reson A 114:39-46.

70. Price WS, Barzykin AV, Hayamizu K, Tachiya M. 1998. A model for diffusive transport through a spherical interface probed by pulsed-field gradient NMR. Biophys J 74:2259-2271.

71. Caprihan A, Wang LZ, Fukushima E. 1996. A multiple-narrow-pulse approximation for restricted diffusion in a time-varying field gradient. J Magn Reson A 118:94-102.

72. Callaghan PT. 1997. A simple matrix formalism for spin echo analysis of restricted diffusion under generalized gradient waveforms. J Magn Reson 129: 74-84.

73. Callaghan PT, Codd SL. 1998. Generalized calculation of NMR imaging Edge effects arising from restricted diffusion in porous media. Magn Reson Imag 16:471-478.

74. Sukstanskii AL, Yablonskiy DA. 2002. Effects of restricted diffusion on MR signal formation. J Magn Reson 157:92-105.

75. Barzykin AV. 1998. Exact solution of the TorreyBloch equation for a spin echo in restricted geometries. Phys Rev B 58:14171-14174.

76. Barzykin AV. 1999. Theory of spin echo in restricted geometries under a step-wise gradient pulse sequence. J Magn Reson 139:342-353. 
77. Niendorf T, Dijkhuizen RM, Norris DG, van Lookeren Campagne M, Nicolay K. 1996. Biexponential diffusion attenuation in various states of brain tissue: implications for diffusion-weighted imaging. Magn Reson Med 36:847-857.

78. Mulkern RV, Gudbjartsson H, Westin CF, Zengingonul HP, Gartner W, Guttmann CR, et al. 1999. Multicomponent apparent diffusion coefficients in human brain. NMR Biomed 12:51-62.

79. Clark CA, Le Bihan D. 2000. Water diffusion compartmentation and anisotropy at high $\mathrm{b}$ values in the human brain. Magn Reson Med 44:852-859.

80. Sehy JV, Ackerman JJH, Neil JJ. 2002. Evidence that both fast and slow water ADC components arise from intracellular space. Magn Reson Med 48: 765-770.

81. Ababneh Z, Beloeil H, Berde CB, Gambarota G, Maier SE, Mulkern RV. 2005. Biexponential parameterization of diffusion and $\mathrm{T} 2$ relaxation decay curves in a rat muscle edema model: decay curve components and water compartments. Magn Reson Med 54: 524-531.

82. Milne ML, Conradi MS. 2009. Multi-exponential signal decay from diffusion in a single compartment. J Magn Reson 197:87-90.

83. Callaghan PT. 1995. Pulsed gradient spin echo NMR for planar, cylindrical and spherical pores under conditions of wall relaxation. J Magn Reson A 113:5359.

84. Söderman O, Jönsson B. 1995. Restricted diffusion in cylindrical geometry. J Magn Reson A 117:94-97.
85. Sen PN, Hürlimann MD, de Swiet TM. 1995. DebyePorod law of diffraction for diffusion in porous media. Phys Rev B 51:601-604.

86. Frohlich AF, Ostergaard L, Kiselev VG. 2006. Effect of impermeable boundaries on diffusion-attenuated MR signal. J Magn Reson 179:223-233.

87. Bennett KM, Schmainda KM, Bennett R, Rowe DB, Lu H, Hyde JS. 2003. Characterization of continuously distributed cortical water diffusion fates with a stretched-exponential model. Magn Reson Med 50: 727-734.

88. Peyron M, Pierens GK, Lucas AJ, Hall LD, Stewart RC. 1996. The modified stretched-exponential model for characterization of NMR relaxation in porous media. J Magn Reson A 118:214-220

\section{BIOGRAPHIES}

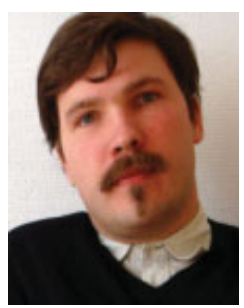

Denis S. Grebenkov has received two Ph.D. degrees, the first in statistical physics from Saint Petersburg State University (Russia), in 2003; and the second in theoretical physics from Ecole Polytechnique (France), in 2004. He did postgraduate researches on lung imaging at University Paris-XI (France), and on nonequilibrium dynamics of granular media at University of Naples (Italy). In 2006, he got a permanent research position at Laboratory of Condensed Matter Physics, CNRS, Ecole Polytechnique (France), to investigate restricted diffusion in complex geometries. 